\title{
Posttraumatic carotid-cavernous fistula - an endovascular carotid stent-graft approach
}

\author{
Ionut Flavius Bratu, Athena Cristina Ribigan¹,2, Bogdan Dorobat', Vlad Eugen Tiu², \\ Bogdan Casaru' ${ }^{2}$, Ovidiu-Alexandru Bajenaru ${ }^{1,2}$, Florina Anca Antochi ${ }^{2}$ \\ 1"Carol Davila" University of Medicine and Pharmacy, Bucharest, Romania \\ 2Department of Neurology, Emergency University Hospital, Bucharest, Romania \\ ${ }^{3}$ Department of Interventional Neuro-Radiology, Emergency University Hospital, Bucharest, Romania
}

\begin{abstract}
Objectives. The carotid-cavernous fistula (CCF) represents an abnormal communication developed between the internal and/or the external carotid artery (ICA, ECA) or their branches and the cavernous sinus (CS). We report this case in order to highlight the course and an alternative to treatment of a CCF.

Material and methods. A 47-year-old male patient with medical history of sub-arachnoid hemorrhage and craniofacial comminuted fractures due to a motorcycle accident-induced cranio-facial traumatism (4 months prior to admission) was referred to our clinic for further investigation regarding a potential CCF.

Results. The cerebral digital subtraction angiography showed a direct CCF type A. The Neurology and Interventional Radiology team opted for endovascular stent-grafting of the right ICA.

Conclusions. This case is presented in order to highlight that angioplasty with stent-graft is a safe and effective procedure in selected cases of CCF.
\end{abstract}

Keywords: carotid-cavernous fistula, endovascular, stent-graft

\begin{abstract}
Abbreviations list:
$\mathrm{CCF}(\mathrm{s})=$ carotid-cavernous fistula(e);

$\mathrm{CS}=$ cavernous sinus;

ICA = internal carotid artery;

$\mathrm{ECA}=$ external carotid artery;

$\mathrm{CT}=$ computed tomography;

$\mathrm{F}=$ French .
\end{abstract}

\section{INTRODUCTION}

The carotid-cavernous fistula represents an abnormal arterio-venous communication developed between the internal and/or the external carotid artery or their branches and the cavernous sinus. The most widely used criteria for the classification of CCFs are: anatomy (angiographical criterion), etiopathogenesis and blood flow (1).

Based on the anatomical-angiographical criterion, according to Barrow and Tomsick, the CCFs can be classified as direct (intracavernous segment of the ICA and CS direct connection) and indirect or dural (communication via branches of ICA and/ or ECA). Furthermore, the classification involves five types: type A (direct connection between the intracavernous segment of the ICA and the CS), type B (dural communications between intracavernous branches of the ICA and the CS), type C (dural communications between meningeal branches of the ECA and the CS) and type D (dural communications between the intracavernous branches of the ICA, meningeal branches of the ECA and the CS) with the subtypes D1 and D2 based on the arterial supply being unilateral or bilateral $(2,3)$.

As for their etiopathogenesis, the CCFs can be divided into posttraumatic, which account for 70$90 \%$ of the CCF cases and other etiologies, including spontaneous (4). 
The posttraumatic CCFs usually develop due to patients being involved in car accidents, falling or suffering penetrating cervical-cerebral trauma (5), only a minority of the posttraumatic CCFs $(0,1 \%)$ (4) being a result of iatrogenic trauma - transsphenoidal surgery for pituitary pathology, rhinoplasty, rhizotomy, embolization of meningeal tumors (5). The hypothesized mechanisms for the development of posttraumatic CCFs range from arterial wall pierce due to cranial fracture fragments to exponential rise in the shearing forces and/or of the local intraluminal blood pressure as of the abrupt and forceful flexion of the cervical region (6). Although there have been identified cases of posttraumatic indirect CCFs, the majority of the posttraumatic CCFs are direct, type A CCFs (5). Most of the posttraumatic CCFs are unilateral, only in $1-2 \%$ of the reported cases the CCFs being present on both sides (7).

As of the blood flow the CCFs are classified as low-flow (indirect CCFs) and high-flow (direct CCFs) (1).

The signs and symptoms exhibited by the patients with CCFs vary because of parameters such as the dimensions of the fistula, its location, the anatomical and histological properties of the implicated blood vessels, length of the evolution of the fistula and the venous drainage, but the most important variable influencing the clinical features is the blood-flow $(8,9)$.

The most frequent clinical features of direct CCFs are ocular and neurological and they include: headache, ocular and/or orbital pain, ocular bruit, chemosis, proptosis, secondary glaucoma, reduced eyesight, cranial nerve palsies (III, IV, VI nerves) and sensory abnormalities in the facial territories innervated by the first and/or the second branches of the fifth cranial nerve (10-12). Clasically, the posttraumatic CCFs have an onset presentation known as Dandy's triad (8): proptosis, chemosis and bruit.

The positive diagnosis is ultimately reached by means of medical imaging, digital subtraction angiography being the gold-standard in diagnosing and treating CCFs (13).

\section{CASE REPORT}

A 47-year old male patient with history of cranio-cerebral trauma (4 months before his admission) and left eye childhood posttraumatic atrophy was referred to our Neurology Department for fur- ther investigation regarding a potential carotid-cavernous fistula. 4 months prior to his admission in our clinic, the patient was brought to the Emergency Room of another hospital following a motorcycle accident, exhibiting right ear otorrhagia, headache and sleepiness. The cerebral CT scan showed a posttraumatic right temporal intracerebral hematoma with subarachnoid hemorrhage and cerebral oedema, right temporo-parieto-occipital and nasal pyramid comminuted fractures. The patient was released 3 weeks later, a subsequent CT-scan having shown improvement of the cranio-cerebral lesions.

3 months after discharge the patient was referred to the Ophthalmology Clinic for right eye exophthalmos, permanent bruit in the right ear and severe chemosis. In this clinic, the ophthalmological examination established the following features of the right eye: presence of intractable, painless exophthalmos, elevated intraocular pressure, severe chemosis, herniation of the inferior conjunctiva, lens opacities, corneal abrasions and limited eye movement in all directions, symptomatology that raised the suspicion of a carotid-cavernous fistula. Anti-inflammatory, analgesic and specific glaucoma treatment was recommended and the patient was referred to our clinic for further investigation of the potential CCF.

The general physical exam was normal. The neurology examination established the presence of severe hyposmia, exophthalmos and limited abduction of the right eye, corneal leukoma and blindness of the left eye and a mild peripheral vision loss in the right eye. The patient was oriented to time and space.

The blood tests revealed only mild inflammatory syndrome and dyslipidemia.

Three ophthalmology examinations where performed - two before and one after the treatment of the CCF. The first one accounted for the following features of the right eye: perception of hand movement, chemosis and conjunctival hyperaemia (Fig. 1), post central retinal vein occlusion status, neovascular glaucoma (26 $\mathrm{mmHg}$ pressure under Timolol), axial exophthalmos, altered oculomotricity in the external quadrant, superficial punctate queratitis, deep asymmetrical anterior chamber, posterior synechiae of the iris, rubeosis iridis and the presence of pigment deposits on the anterior capsule of the lens. As for the left eye - the examination noted the lack of light perception, total corneal leukoma and disorganized eye bulb; the intraocular pressure 
couldn't be measured. Right eye fundoscopy revealed blurred optic disc margins, dilated blood vessels, thickened, tortuous and sinuous veins, flame shaped retinal hemorrhages and macular neovascularization with hardly distinguishable details. A combination of ophthalmic drops was administered for lowering the intraocular pressure, alleviating ocular and surrounding tissue inflammation and antibiotic prophylaxis. The follow-up ophthalmology examination revealed slight improvement in the visual acuity of the right eye and intraocular pressure dropping down to $20 \mathrm{mmHg}$.

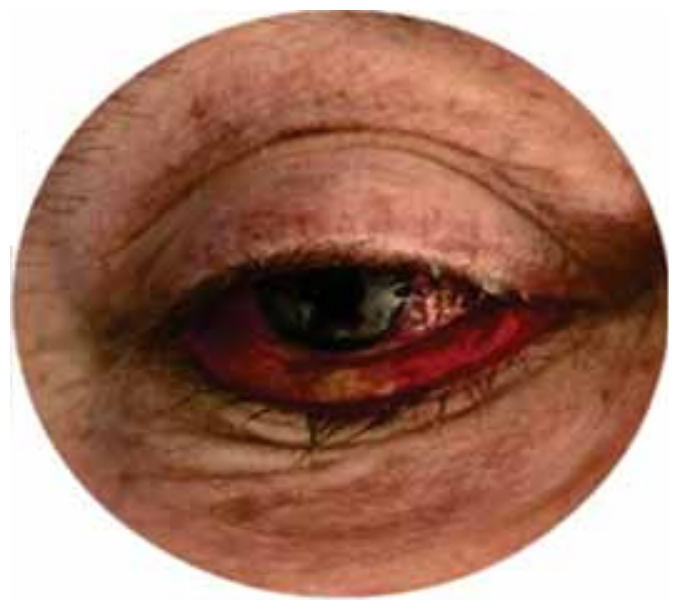

FIGURE 1. Exophthalmos, chemosis and conjunctival hyperaemia of the right eye.

Cerebral digital substraction angiography was performed using a $5 \mathrm{~F}$ sheath and the selective injection of the ICA, ECA and the vertebral artery (on both sides) revealed the presence of a direct CCF (Fig. 2), type A, the affluent being the intracavernous segment of the ICA. Its venous drainage was through an extremely dilated, tortuous ophthalmic vein to the right facial vein.

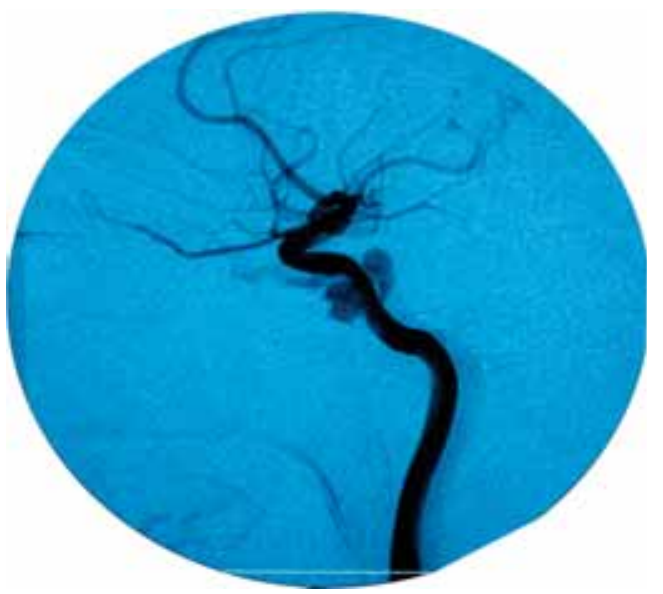

FIGURE 2. The cerebral digital substraction angiography - sagittal plane - showing the presence of a right direct carotid-cavernous fistula.
As there were no contraindications, the Neurology and Interventional Radiology team established that the appropriate course would be interventional treatment. Therefore, stent-graft angioplasty was done through the right femoral artery using a $7 \mathrm{~F}$ sheath and a $7 \mathrm{~F}$ guide catheter. After a $7 \mathrm{~F}$ guiding sheath was placed in the lacerum segment of the ICA, angioplasty was done placing a $5 \times 18 \mathrm{~mm}$ Bentley stent-graft (BeGraff) into the intracavernous segment of the ICA (origin of the CCF), using a balloon inflated at 8 atmospheres. Following the angioplasty, the angiography showed no evidence of CCF patency (Fig. 3). During the procedure, $5000 \mathrm{UI}$ of heparin and $1 \mathrm{ml}$ of nitroglycerin were administered as part of the intraprocedural prophylaxis protocol for thromboembolic events and vasospasm. There were no periprocedural events.
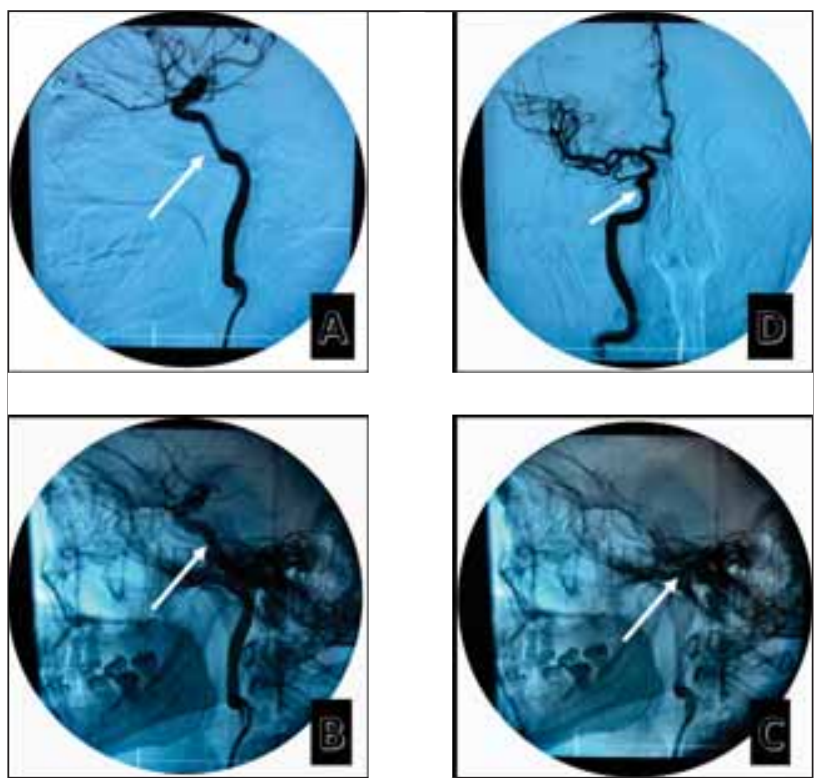

FIGURE 3. The cerebral digital substraction angiography - sagittal $(A, B, C)$ and coronal planes $(D)$, taken following the angioplasty, showen no evidence of carotid-cavernous fistula patency.

As for the use of the stent-graft double antiplatelet therapy with aspirin and clopidogrel was recommended. The patient also received treatment with atorvastatin for dyslipidemia.

The ophthalmology examination performed after the endovascular procedure established the significant improvement of the eye-sight deficit, deep asymmetrical anterior chamber, posterior synechiae of the iris, rubeosis iridis and mild central corneal oedema of the right eye. The right-sided intraocular pressure was still $20 \mathrm{mmHg}$. The optical coherence tomography revealed no abnormalities 


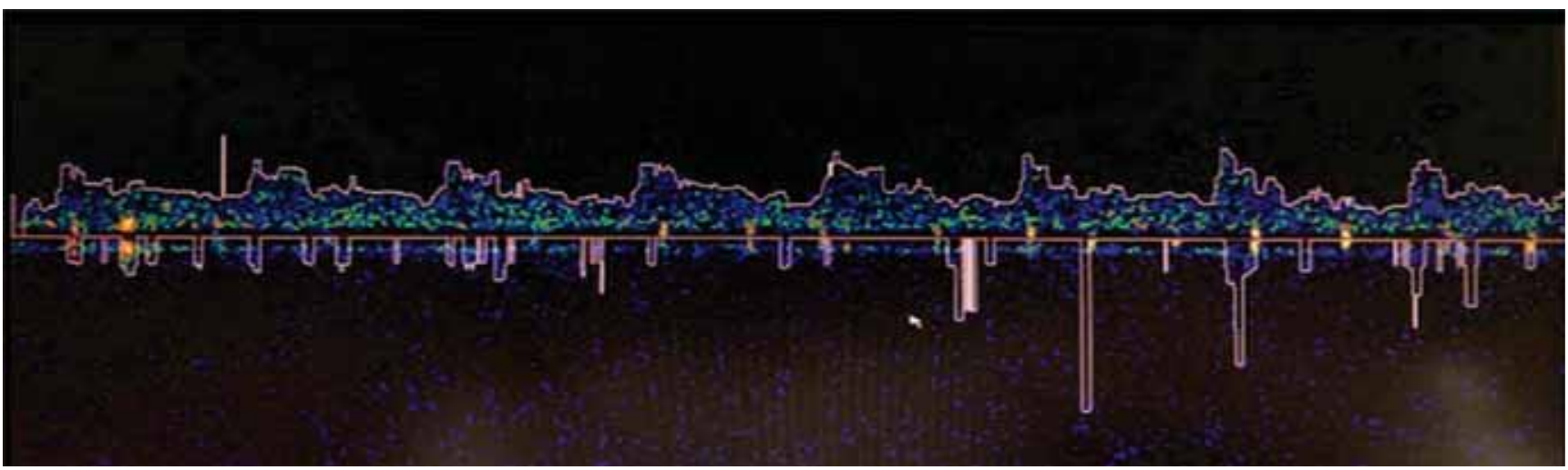

FIGURA 4. The transcranial Doppler ultrasonographic examination of the intracranial arteries showing the patency of the carotid stent-graft and normal velocimetry.

of the optic nerve and macula. The ophthalmologist recommendation was for the patient to continue administering the moisturizing and glaucoma ophthalmic drops and to use artificial tears until further evaluation at one month.

The post-procedural follow-up transcranial Doppler ultrasonographic examination of the intracranial arteries showed the patency of the carotid stent-graft and normal velocimetry (Fig. 4).

The patient was discharged from our clinic 2 weeks after his admittance with the recommendations mentioned above.

Follow-up consisting of neurology, ophthalmology examinations and transcranial Doppler ultrasonography was scheduled at 1-3-6-9-12 months and after that, annually.

\section{DISCUSSIONS}

The management of CCFs includes monitoring the ophthalmological status, closing the fistula and treating its complications, all being done while taking into account the type of fistula, the vascular architecture, the signs and symptoms and the associated neurological and ophthalmological risks in terms of morbidity and death (1).

The aim of the endovascular management is closing the CCF while preserving the patency of the carotid artery. Obliterating the fistula and its associated pressure-generated changes determines the remission of the signs and symptoms. The advantages of the endovascular treatment include the fact that is minimal invasive, it doesn't require general anesthesia, the postprocedural recovery time is greatly reduced and it has a low associated morbidity rate compared to other types of treatment (14).
The rate of success of endovascular treatment is 55$99 \%$ with an associated complications rate of $10-$ $40 \%$ (ICA occlusion, cerebral infarction, worsening of the cranial nerves paresis) (9).

Transarterial embolization is the preferred method in treating the majority of CCFs. It uses platinum coils, detachable silicone or latex balloons or liquid adhesive agents such as Onyx by themselves or in association with a stent (15).

Another viable option of endovascular management reported in literature is the use of stent-grafts for closing the posttraumatic CCFs and preserving the patency of the ICA, particularly in those patients who presented low tolerance to the balloon occlusion test used during the diagnostic angiography to evaluate the collateral circulation stemming from the circle of Willis and how the patient would react to his obliteration of the ICA (16).

The main disadvantages of using a stent-graft include low flexibility making endovascular transport and placement difficult through the sinuous and tortuous cerebrovascular network and the periprocedural vasospasm developed because of the vascular irritation caused by/at the ends of the stent-graft (17). There is a lack of studies on longterm safety of using stent-grafts in treating CCFs.

\section{CONCLUSIONS}

This case was chosen to be presented in order to highlight that endovascular stent-grafting is a safe and effective procedure in selected cases of carotid-cavernous fistulae and that appropriate management sustained by a multidisciplinary team may reduce the associated risks of morbidity and death. 


\section{REFERENCES}

1. Scott IU. Carotid-Cavernous Fistula (CCF): Background, Pathophysiology, Epidemiology [Internet].

2. Barrow DL, Spector RH, Braun IF. Classification and treatment of spontaneous carotid-cavernous sinus fistulas. J Neurosurg. 1985; 62(2):248-56.

3. Ringer AJ, Salud L, Tomsick TA. Carotid cavernous fistulas: Anatomy, classification, and treatment. Vol. 16, Neurosurgery Clinics of North America. 2005. p. 279-95.

4. Lin T-C, Mao S-H, Chen C-H et al. Systematic Analysis of the Risk Factors Affecting the Recurrence of Traumatic Carotid-Cavernous Sinus Fistula. World Neurosurg. 2016;90:539-545.e1.

5. Mergeani A, Popescu D, Laza C et al. A review on endovascular techniques for treatment of direct post-traumatic carotid-cavernous fistula supported by case presentations. Mædica. 2012;7(4):332-8.

6. Coskun O, Hamon M, Catroux $\mathrm{G}$ et al. Carotid-cavernous fistulas: Diagnosis with spiral CT angiography. Am J Neuroradiol. 2000; 21(4):712-6.

7. Gemmete JJ, Ansari SA, Gandhi DM. Endovascular Techniques for Treatment of Carotid-Cavernous Fistula. J Neuro-Ophthalmology. 2009;29(1):62-71.

8. Korkmazer B, Kocak B, Tureci E et al. Endovascular treatment of carotid cavernous sinus fistula: A systematic review. World J Radiol. 2013;5(4):143-55.

9. Bennett JL, Gonzalez MO, Durairaj V. Carotid-cavernous fistulas - UpToDate [Internet].
10. Ono K, Oishi H, Tanoue S et al. Direct carotid-cavernous fistulas occurring during neurointerventional procedures. Interv Neuroradiol. 2016;22(1):91-6.

11. Kupersmith MJ, Berenstein A, Flamm E et al. Neuroophthalmologic Abnormalities and Intravascular Therapy of Traumatic Carotid Cavernous Fistulas. Ophthalmology. 1986;93(7):906-12.

12. Wang W, Li YD, Li MH et al. Endovascular treatment of post-traumatic direct carotid-cavernous fistulas: A single-center experience. J Clin Neurosci. 2011;18(1):24-8.

13. Shownkeen H, Bova D, Origitano TC et al. Carotid-cavernous fistulas: pathogenesis and routes of approach to endovascular treatment. Skull Base. 2001;11(3):207-18.

14. Amuluru K, Al-Mufti F, Gandhi CD et al. Direct carotid-cavernous fistula: A complication of, and treatment with, flow diversion. Interv Neuroradiol. 2016;22(5):569-76.

15. Ng PP, Higashida RT, Cullen S et al. Endovascular strategies for carotid cavernous and intracerebral dural arteriovenous fistulas. Neurosurg Focus. 2003;15(4):ECP1.

16. Vattoth S, Cherian J, Pandey T. Magnetic resonance angiographic demonstration of carotid-cavernous fistula using elliptical centric time resolved imaging of contrast kinetics (EC-TRICKS). Magn Reson Imaging. 2007;25(8):1227-31.

17. Turner RD, Gonugunta V, Kelly ME et al. Marginal Sinus Arteriovenous Fistulas Mimicking Carotid Cavernous Fistulas: Diagnostic and Therapeutic Considerations. Am J Neuroradiol. 2007;28(10):1915-8. 